\title{
An Image of Aging People's Life Expectations with a Focus on Iran
}

\author{
Mohammad Taghi Sheykhi \\ Department of Social Science, Alzahra University, Tehran, Iran \\ Email address \\ mtshykhi@alzahra.ac.ir, mtshykhi@yahoo.com
}

\section{To cite this article:}

Mohammad Taghi Sheykhi. An Image of Aging People's Life Expectations with a Focus on Iran. Humanities and Social Sciences. Vol. 7, No. 3, 2019, pp. 83-88. doi: 10.11648/j.hss.20190703.11

Received: January 22, 2019; Accepted: April 12, 2019; Published: June 24, 2019

\begin{abstract}
The research investigates how due to technological change and medical improvement since mid 20th century people's life course has expanded, followed by new social, economic and medical expectations. People in their late sixties are expecting higher standards and improved quality of life as compared with previous generations. Similarly, because of adoption of new standards including lower birth rates within the generations in their fertility life course, increasing aging is expected in the decades to come. Under such circumstances, appropriate plans and policies need you be executed to expand the emerging expectations of the elderly people. The elderly people's social security and pension system need to be modified and improved. In the absence of which the aging people will remain poor and in an uneasy quality of life.
\end{abstract}

Keywords: Technological Change, Expanding Life Expectancy, New Expectations, Plans \& Policies, Quality of Life

\section{Introduction}

Sociologically and medically speaking, increasing age is inevitably associated with increased exposure to risk factors and reduced adaptability which results in disease and loss of health. In the meantime, and in sociological context, protective influences such as wealth, status and social networks are highly responsible for health-related issues and quality of life of the elderly not only in Iran, but in many other countries of the world. Health and social policy play a determining role in resolving health-related issues in Iran.

While years are automatically added to life due to progress in various sectors of socio-economic life, appropriate and adequate measures should be taken so far as the elderly's quality of life is concerned [1]. This means that we are more interested in how long people live in a healthy state and are less interested in simply prolonging life when severe disability has taken hold. However, concerns are usually raised about the way in which technological advances in medical practice have resulted in people being kept alive despite terrible impairments of communications, mobility and pain.

Population aging seeks to explore various objectives with special reference to social development and public health as a result of increase in global life expectancy. The paper will find out how aging in modern times is increasingly becoming a norm rather than the exception of a few in more affluent societies. Similarly, one of the ultimate purposes of the present paper is to display and promote health and wellbeing, and thus ensure the attainment of the best quality of life for as long as possible, and for the increasing number of the elderly in Iran.

Indeed, the paper seeks to find out how to prevent and / or deal with problems commonly associated with aging. However, for some, old age is accompanied by frailty, disease and disability; each year millions of elderly people survive in poor health and dependency around the world as well as in Iran.

The paper will identify and demonstrate the determinants of aging of population which are mainly due to reduction in fertility and death rates at all ages. It is worth mentioning that in case of Iran in early 21 st century, more reduction in death rates contribute to aging. However, over the more recent past, development in medical care has considerably contributed to gains in life expectancy.

Under the conditions that the aging population in Iran is ever on rise, i.e. above 8 percent of the total population of 81.6 million in 2018 [2], yet the ratio is even higher in capital city of Tehran as compared with other metropolitan areas. Similarly, while in 2002 the population of the province of 
Tehran was estimated 11.7 million, that of greater Tehran city was estimated 7.1 million, out of which 5.2 percent was estimated aged 65 and above. Likewise, since Tehran is changing into a megacity with relevant social, psychological, economic and cultural features of megacities, coming to know the phenomenon of aging, protecting elderly and raising their quality of life is the priority of the research.

The paper also aims to appraise the pathological aspects of the aging population, their needs, and services provided to them. Although the policy-makers and social researchers are themselves prone to the problems of aging, they do not much think about their future and the uncertain loneliness [3]. Since Iran's life expectancy has almost doubled during the 20th century; reaching 69 years in 2003, followed by over 75 in 2018 [4], it has created new socio-cultural and health problems in the country and within the elderly.

Changes in family size and structure are also generating new challenges and health-related issues for the elderly. The traditional joint / extended family is being superseded by the nuclear family; giving rise to various health issues including the mental health concerns of the elderly, previously not common. The phenomenon is not only appearing for the Iranian society, but the situation is the same in the Indian society too. However, the Iranian elderly are more vulnerable due to social and economic marginalization. For the poor, old age is a curse, and it is worse to be an old woman than an old man [5]. They become an additional burden on the already fragile social security system of the country as well as the family under the conditions of extensive unemployment.

\section{Research Methodology}

The research methodology used in the present paper is a combined form of theoretical and empirical methods. In that, the researcher first initiated probing into the sociological and demographic literature of the elderly, to be prepared to develop empirical enquiries to collect the relevant data. In the theoretical part, the author has tried to use various sociological and gerontological theories. Subsequently, the research was followed by administering one-hundred questionnaires, accompanied by interviews where necessary. Eventually, 80 percent of the questionnaires were extracted and analyzed.

\section{Conceptual Analysis}

It is really difficult to make comparisons with attitudes in the past since so little is known about aging except what is recorded in fiction [6]. The more we go to the past, the more respects were paid to the elderly not only in Iran, but in every other society. That is to say, the type of social structure and family structure contributed to more respects and esteem for the seniors who were also very influential within the family and the community as a whole. They were looked upon as sources of all knowledge and repositories of great wisdom. Since time and conditions have changed, they are not looked upon as before due to several reasons. Under such conditions children were much less likely to have had a grandparent alive in the 19th century, or even early 20th century, and hence the latter had scarcity value. Older people were also likely to have had comparatively greater skills and knowledge, since changes were little from generation to generation. In contrast, today due to rapid increase in knowledge, and changes in technology, it is difficult for one generation to keep up with the next.

The health status of older persons in Iran as everywhere else, is a factor of central importance for a number of reasons. At the individual level the capacity to earn a living or to participate in family and community life, as well as a sense of personal well-being, are all governed to a great extent by health status. Despite the critical importance of health care, both the extent and accessibility of health services and special medical care for older people remain very limited in many developing countries including Iran. For example, health facilities and personnel tend to be concentrated in urban areas, while older people are still predominantly rural dwellers throughout much of the developing countries. Access to affordable health care has been identified as a major challenge for the developing countries' health by the turn of the century. In the absence of comprehensive public health care, older people adopt a number of different strategies [7]. The absence of accessible and affordable health facilities leads many older people to adopt self-help strategies such as buying imported medicine without prescription, or even in many occasions, they have to put up with ills.

Though growing old in one's own community is the overwhelming preference for most people, yet, many old people are deprived of that. In Iran as well as in many other parts of Asia, there is a strong consensus on the importance of the family as a basis component of the social structure, and as a result, a key role is assigned to families is that of providers of care at every stage of life with a focus on the elderly. However, the rapid demographic transition of recent decades, accompanied by other changes such as migration, urbanization, and the increasing numbers of women entering the formal workforce, all have deteriorated the conditions of the elderly in Iran.

The physical changes that come with age are varied and do not necessarily develop at the same time in each old person. It is useful to have some understanding of these physical and mental changes, and to know what is normal and what is abnormal. So far as the health of the elderly people is concerned, unfortunately many of the public still feel that little can be done for an elderly man or woman who is ill, i. e. it is assumed that the old person's condition is simply due to his/her age. In fact, most old people are suffering from a treatable disease of one sort or another [8].

In terms of demographics, more attention must now be focused on older people since their numbers are increasing rapidly. Such people must come under more protection of medical cares, better food, protection etc. However, the assumption that "the elderly" are a social problem, or even a burden, that they have similar aspirations, and that they all 
need certain services, all are questionable.

Some thinkers illustrate that aging is a development issue. The growth of older populations however, poses a challenge to the system of health care [9]. As a person grows older, his / her physical function deteriorates. There are several characteristics of illness in order people as a consequence of age-associated loss of biological adaptability, multiple pathology, the need for rehabilitation etc.

However, healthy older persons are a resource for their families, their communities and the economy. To reach the goal of a society that all ages share together, we must improve our system of health services. This must be done for the coming society of aging populations, and to ensure that all of them enjoy the highest attainable level of well-being, as called for by Dr. Nakajima, the former Director-General of WHO.

Based on a definition, aging is known to be the third stage of life; after dependency as the first, followed by the second stage as the time of independence in which maturity, taking responsibility and making income and savings take place. The third stage is also known as redependency [10]. Similarly, concepts such as social class, family protection, mental \& physical ability and occupational patterns - all count in differentiation within the elderly people.

Statistics on life expectancy at birth obscure the long life span that many women in developing countries already have beyond age 50. The majority of the world's older women are already living in developing countries including Iran. Although the economic, social, political and cultural contexts which shape their lives are vastly different from those in developed countries, the transformation of their roles is occurring much more rapidly than in the older industrialized countries. However, women's extended life span calls for attention to the quality of life in later years in all societies.

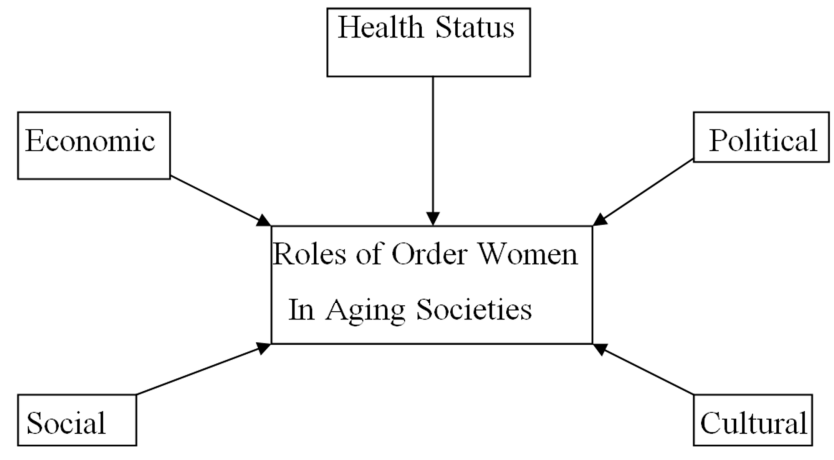

Figure 1. General Determinants of Roles of Older Women.

Widows comprise the largest group of aging people in almost every country including Iran. In case of Iran, a large number of widows remain unremarried for the whole of their lives more due to the socio-cultural values present in their societies, cultural barriers etc.

Comparative statistics show a contrasting variation in the proportion of the elderly people in industrialized and developing societies. While 13 percent of people over the age of 65 belongs to the developed world, the same proportion averages about 4.5 per cent in the developing world [11].

Table 1. Trends in Life Expectancy From Age 65 in Selected Years in Developed World (\%).

\begin{tabular}{lll}
\hline Year & Men & Women \\
\hline 1976 & 12.5 & 16.6 \\
1981 & 13.1 & 17.1 \\
1985 & 13.4 & 17.3 \\
1988 & 13.7 & 17.6 \\
1991 & 14.2 & 17.9 \\
\hline
\end{tabular}

Source: Aging and Health, WHO Symposium, 1998.

\section{Profiles and Challenges of Aging}

The total population of Iran is currently over 81.6 million, and is expected to reach 84.7 million in the year 2025. Under such population trends, the number of the elderly people in need of long-term care due to infirmities, dementia, and other conditions, will also sharply increase. Such a demographic change will create social security needs including pensions, medical services, welfare services and the like.

Decline in fertility and mortality rates in Iran make for a gradual process of aging society. If the relevant institutions do not respond to the needs of the elderly, the emergence of challenges are inevitable.

Sociologically speaking, in all likelihood, as today's adults grow old, the elderly of the future will demand more from society than do the elderly populations of today. Future generations will be better educated, and today's adults will demand more in their old age to maintain the lifestyles to which they have been accustomed [12].

Such momentum of demand has implications for the current allocation of resources for the elderly of a country like Iran. In that, the society has to tolerate various pressures coming from having to provide for the young as well as the adults in an economy characterized by poverty, unemployment and inequalities. However, the quality of life and the status of the elderly in such a society are the byproducts of their socio-economic position prior to reaching the old age threshold.

The scenario of aging and health is further complicated due to various reasons such as inadequacy of current information on their number, their living conditions, as well as the lack of multidisciplinary research to address policy development and intervention needs.

Worldwide, population aging being one of the major achievements of the 20th century, is mainly caused by an interaction of factors such as improvements in nutrition, education, hygiene, health care and living standards. Although in the less developed countries like Iran, the proportions of the elderly are still relatively low, but the process of aging is occurring at an increasingly rapid pace. Since developing countries including Iran have fewer financial resources, the social and economic impacts of which on elderly societies will be much higher and more difficult to handle; hence careful planning is required [13].

In the 21 st century, aging in the new world will be 
experienced as never before. The age structure of the world will continue to change - by the fertility continuing to decrease towards the population replacement level, and consequently life expectancy increasing in every region of the world. However, though the industrialized countries have by far the oldest age structure, i.e. more than 15 percent, soon the developing countries including Iran will reach the same situation.

\section{Healthy Life Perspectives}

Concerning the health of the elderly, much emphasis has to be placed on the concept of "healthy aging". This concept is based on the notion that the lifestyle of an individual together with the environment can greatly influence life expectancy and health. Many countries currently have health promotion programs (such as stimulation of physical exercise, healthy nutrition and prevention of injury) aimed at older persons [14].

Nowadays, people live longer than ever before, and it is mainly higher quality of life that contributes to that. There rises a question here; and that is: Are people also living a healthier and disability-free old age, or they spend a longer share of their life in ill-health? To answer this important question, the concept of "healthy" or "disability-free" life expectancy has been developed. This concept is important to policy-makers since the longer the elderly stay in good health and free from disability, the smaller the cost for the health care and social services will be.

Healthy life expectancy depends on a few chronic diseases that influence disability with increasing age, such as circulatory diseases, mental disorders e. g. Alzheimer's disease, arthritis and the like. Since many elderly become frail and experience poor health when they are over the age of about 80 years (in many developing countries about 70 years), the definition of old age (people who are no longer economically active) is called into question. Indeed, most persons in their $60 \mathrm{~s}$ or $70 \mathrm{~s}$ have nearly the same health characteristics as the middle-aged population [15]. On the whole, the costs of health care provision increase with age; particularly after the age of 70 in countries like Iran, where the demand is growing.

Several studies indicate that women spend a greater percentage of their old age in a disabled state. That is, the higher life expectancy (both at birth and at age 65) for women is thus partly offset by a lower healthy life expectancy [16]. Furthermore, it has been proved that there is a positive relation between income and education: persons in the poorest and the least educated part of the population have also the lowest health expectancy, and spend a greater part of their life disabled or handicapped [17]. Similarly, health expectancy tends to be higher among married rather than among unmarried persons [18].

However, a transition should take place toward a new culture of old age, including a more dynamic life and continued participation in societal activities. This should also involve changes of attitudes towards the elderly. Although they are no longer economically active, the oldest old deserve respect. While in most traditional communities the elderly have a role of prestige, in a materialistic society they are sometimes considered as a burden. It is to be noted that nuclearization of families tend to isolate the elderly.

\section{Social Distance}

The term refers to the perceived feelings of separation or isolation among different age groups with special reference to the elderly. It is most commonly used to indicate the degree of gap between the members of different age cohorts [19]. However, the concept of social distance or social isolation is not easy to measure. In that, social contacts by the elderly are minimized. Although the elderly may have the ability to give help to others, there may be other constraints which may impede them to do so; and that is where they feel isolated.

The inability to visit relatives and friends, the inability to adopt useful roles, frequent loneliness and discouragement all contribute to isolation among the elderly [20]. However, social isolation is more severe and problematic for the women since they live longer than their husbands. That is why they are more prone to psychological and mental damages. Retirement, loss of prestige and credits, loss of social roles and the like contribute to social isolation and social distance.

\section{Assessment of Data}

In this part of the research, the author tends to reflect some of the results/findings of the survey which include the elderly way of life in Tehran, the quality of cares and services provided to them, their satisfaction with life and queries of such kind.

However, as the trend of population aging in Iran like many other developing countries is much faster than the developed countries, to adjust to the phenomenon in various dimensions is not that easy. As a whole, ageism refers to the negative stereotype of elderly individuals, which prejudicially describes them as senile and rigid in their attitudes, and psychologically and socially dependent. In "study population" the author referred to 37 percent men and 63 percent women, and the highest percentage of age groups, i.e. 25 percent belonged to the age range 75-79.

On the other hand, since men have more possessions as compared with women in the Iranian society, they usually remarry after losing their spouses. Under such conditions, they have more chances to live with their children, to be protected by them, and thereby to have better health and cares.

Literacy rate was found 45 percent among the male samples and 55 percent among the female samples. That contributes much to the elderly well-being in our "study population". Similarly, the frequency of literacy among women 80 years old and above was found much more than men. 
To indicate the economic status of the elderly, the author came to know that more than 30 percent of the samples mainly depended on their children's assistance. Similarly, 23 percent of the respondents reported that their main source of income was their pensions, while 22 percent declared that they mainly depended on charities.

Based on findings, 27 percent of the interviewees stated they did not have any health problems, while 65 percent stated that they had some physical disability. Similarly, 6.5 percent reported to have some sort of mental disorder. Overall, and on average, 27 percent of both elderly men and women reported of being in healthy conditions. The researcher also came to know that 10 percent of male respondents suffered from some psychological disability, and that of females was 4 percent.

In another inquiry, 11.5 percent of the respondents reported to have spouse, 52.6 percent declared to have lost spouse due to death of the spouse, 24 percent reported of being alone due to divorce, and finally 11.5 percent of the samples declared as never married. In general, all those without spouse confessed to have more problems.

In visiting the elderly in some care centers, 6.4 percent of the elderly reported to have relations with relatives outside the care centers once a week; that is to say, relatives often come to visit them at the care centers, or the elderly go to visit relatives once a week, while 25.6 percent reported of visits with relatives once a month. Similarly, 56.4 percent of the samples responded that they did not have relations with outside of the care centers at all, eventually 11.5 percent did not react at all.

In a question regarding the reaction of the respondents towards care system / standards in the centers, 66.6 percent assessed it satisfactory, 21.8 percent reflected care standards as medium, while 7.7 percent declare it weak and poor.

In connection with access to medicine, 59 percent of those staying in care centers were well satisfied with medicine provision, 23.1 percent reported the provision was medium, and 7.7 percent of the elderly reported it weak. Eventually, 10.3 percent did not declare at all.

As far as the food / catering is concerned, 64 percent of the elderly in care centers were well satisfied with food conditions, 20.5 percent appraised it medium and 10.3 percent of the elderly found it poor.

\section{Conclusion}

Iran highly needs to have public health programs that encourage healthy habits and life styles to enhance the quality of life of the increasing elderly in the country. A successful health and social service system needs to be evolved which provides all-country access to collaborative care, health education, health promotion and disease prevention.

Based on the motto: the sands are constantly changing, thus, the rapid changes in all spheres of life in the past 70 years mean that older people whose childhood was marked by poverty, a rural background, large families, and a traditional way of life, are now living in modernized urban societies the elderly with new socio-economic expectations. While the process of population aging is fast everywhere including Iran, the phenomenon has a number of negative consequences such as socio-economic, medical, psychological and many others. On the other hand, rising expenditures, additional financial support for the elderly and so forth, are there as realities which need to be met. In the meantime, in recent decades, the aging process has been influenced by the socio-economic development of societies too, particularly at the age of globalization. However, better standards of living, freedom from infectious diseases, and better nutrition, all have contributed to the aging of society. Under such circumstances, it is now the time to seek answers and find solutions to the ever-emerging problems of the elderly in Iran. At the same time, population aging presents enormous opportunities as well as enormous challenges for all societies with special reference to the developing ones such as Iran. Not only Iran is facing the challenges of population aging, but the scenario is spreading all across Asia.

\section{Suggestions}

Not only Iran, but many other developing countries have come to realize the need for appropriate policies to respond to their aging clients which could include:

1 The need for integrated planning and decision-making on health and other social services for the elderly.

2 Promotion of "healthy aging" through education, and information dissemination to modify the elderly's diet, fitness and lifestyles.

3 Initiation and reform of pension provisions.

4 Rectification of attitudes towards the concepts of age, ageism and dependency.

\section{References}

[1] WHO, 1998, World Health Report, Geneva, World Health Organization.

[2] World Population Data Sheet, 2018, Population Reference Bureau, Washington DC.

[3] Shamloo, G. 1983, What is Old Age? Why Do We Get Old? 1st ed. Tehran: Sherkat-e-Sahami Chehr.

[4] Population and Housing Census of Iran 2016, Statistical Center of Iran.

[5] Jamuna, D., 1995 Aging Women in India: A Profile: Research and Development Journal, 1995, 1 (3): 3-14.

[6] Tinker, A. 1997, Older People in Modern Society, P. 8. Longman, London.

[7] WHO, 1999, Aging and Health, Kobe Centre, Ceuterick, Belgium.

[8] Keddie, K. 1978. Action with the Elderly, London.

[9] FU, H. 1998. Implications for Health Services for Aging Populations, quoted by Aging and Health, Kobe Centre, Ceuterick, Belgium. 
[10] Laslett, P. 1998, A Fresh Map of Life: The Emergency of the Third Age, Wedenfeld \& Nicolson, London.

[11] Kinsella, K. 1996, Epidemiology in Old Age, BMJ, London.

[12] Caselli, G. and A. Lopez, Health and Mortality Among the Elderly: Issues for Assessment In: Health and Mortality Among the Elderly Population, Oxford Clarendon Press 1996: $3-21$.

[13] Verhasselt, Y. 1997, Aging in a New World, Quoted by Aging and Health, Kobe Centre, Ceuterich, Belgium.

[14] World Population Aging, 2015 United Nations, New York.

[15] Hickes, P. The Impact of Aging on Public Policy. The OECD Observer, 1996-97, 203: 19-21.
[16] Andrews, G. et al., 1986, Aging in the Western Pacific: A Four-Country Study, Geneva, World Health Organization.

[17] Wilkins, R. et al., Health Expectancy in Canada, Late 1970s, Demographic, Regional and Social Dimensions, American Journal of Public Health, 1983, 73 (9): 1073-1080.

[18] Nault, F. et al., 1996, Socio-economic Conditions of the Married in Canada, Demography, 25 (2): 241-259.

[19] Turner, B. S. et al., 2000, The Penguin Dictionary of Sociology, the 4th ed., P. 322, Penguin Books, London.

[20] Townsend, P., 1986, Ageism and Social Policy, Gower, London. 